\title{
Cortical gating of oropharyngeal sensory stimuli
}

\author{
Karen Wheeler-Hegland ${ }^{1,2}{ }^{*}$, Teresa Pitts ${ }^{1,2}$ and Paul W. Davenport ${ }^{2}$ \\ Brain Rehabilitation Research Center, Malcom Randall Veterans Affairs Medical Center, Gainesville, FL, USA \\ 2 Department of Physiological Sciences, University of Florida, Gainesville, FL, USA
}

\author{
Edited by: \\ Bill J. Yates, \\ University of Pittsburgh, USA \\ Reviewed by: \\ Robert C. Basner, \\ Columbia University, USA \\ Carole L. Marcus, The Children's \\ Hospital of Philadelphia, USA \\ Eric Verin, Rouen University Hospital, \\ France \\ Shaheen Hamdy, \\ University of Manchester, UK \\ *Correspondence: \\ Karen Wheeler-Hegland, \\ Department of Physiological Sciences, \\ University of Florida, Box 100144, HSC, \\ Gainesville, FL 32610, USA. \\ e-mail:kwheeler@ufl.edu
}

\begin{abstract}
Somatosensory evoked potentials provide a measure of cortical neuronal activation in response to various types of sensory stimuli. In order to prevent flooding of the cortex with redundant information various sensory stimuli are gated cortically such that response to stimulus 2 (S2) is significantly reduced in amplitude compared to stimulus 1 (S1). Upper airway protective mechanisms, such as swallowing and cough, are dependent on sensory input for triggering and modifying their motor output. Thus, it was hypothesized that central neural gating would be absent for paired-air puff stimuli applied to the oropharynx. Twenty-three healthy adults (18-35 years) served as research participants. Pharyngeal sensory evoked potentials (PSEPs) were measured via 32-electrode cap (10-20 system) connected to SynAmps ${ }^{2}$ Neuroscan EEG System. Pairedpulse air puffs were delivered with an inter-stimulus interval of $500 \mathrm{~ms}$ to the oropharynx using a thin polyethylene tube connected to a flexible laryngoscope. Data were analyzed using descriptive statistics and a repeated measures analysis of variance. There were no significant differences found for the amplitudes S1 and S2 for any of the four component PSEP peaks. Mean gating ratios were above 0.90 for each peak. Results supports our hypothesis that sensory central neural gating would be absent for component PSEP peaks with paired-pulse stimuli delivered to the oropharynx. This may be related to the need for constant sensory monitoring necessary for adequate airway protection associated with swallowing and coughing.
\end{abstract}

Keywords: sensory evoked potential, sensory gating, cough, swallowing, pharynx

\section{INTRODUCTION}

Multiple airway defense reflexes share anatomic substrates contained within the upper airway, including the larynx, pharynx, oral, and nasal cavities. These structures serve as conduits for air and/or food as they participate in the life sustaining functions of respiration and deglutition. The anatomic arrangement of these structures is such that degradation in sensory innervation can impact the generation of precise motor patterns required to swallow or cough safely (Aviv et al., 1997; Addington et al., 1999; Smith Hammond et al., 2001; Teismann et al., 2007) and may lead to uncompensated penetration or aspiration of material to the larynx or lower airways, thereby compromising respiratory function. The timely initiation of a swallow is integral to preventing material from entering the laryngeal vestibule, and the initiation of coughing is integral to ejecting material that may have strayed into the larynx or trachea. Both swallow and cough are dependent upon effective sensory input. Thus, the study of upper airway sensation is an important component in understanding airway defense mechanisms.

Multiple researchers have studied sensory areas that can elicit or modify swallowing and cough using different sensory modalities, including mechanical, gustatory, and thermal stimuli (Doty, 1951; Storey, 1968; Mori and Sakai, 1972; Boushey et al., 1974; Hwang et al., 1984; Sant'Ambrogio et al., 1986; Bradley, 2000; Sciortino et al., 2003; Theurer et al., 2005; Kitagawa et al., 2009). Specifically, the faucial arches and posterior oropharyngeal wall are implicated in swallow initiation, and the laryngeal mucosa for eliciting cough. We have recently shown that air puff stimuli to the posterior oropharynx can induce the urge-to-cough, and in some cases cough
(Wheeler-Hegland et al., in press). The pharyngeal branch of the glossopharyngeal and vagus nerves supplies sensory innervation to the posterior oropharyngeal wall (Mu and Sanders, 2000). This pharyngeal plexus of nerve fibers (along with the superior laryngeal nerve), when stimulated, can elicit the swallow reflex (Doty, 1951; Kitagawa et al., 2002) or a cough (Storey, 1968; Sant'Ambrogio et al., 1986). In their detailed study of a human oro- and laryngopharynx, Mu and Sanders (2000) identified areas densely populated with sensory nerve endings including the posterior oropharyngeal wall. They hypothesized that the increased number of nerve fibers in the oropharynx compared with other areas was related to the significance of this area for swallow initiation (Mu and Sanders, 2000).

The study of upper airway sensation in awake humans has traditionally been reported in terms of either motor output or verbal confirmation of sensory perception, making it difficult to differentiate motor, speech and language, or cognitive abilities from sensory parameters. Event-related electroencephalography is a method that does not rely on motor or verbal feedback when studying sensation in humans. This method has high temporal resolution and allows for the scalp recording of neuronal activity that is time-locked to a sensory stimulus, yielding the sensory evoked potential. It has been shown that electrical and mechanical stimuli applied to the lips, buccal cavities, tongue, faucial arches, soft palate, nasal cavity, and nasopharynx will evoke a cortical sensory response (Fujiu et al., 1994; Maloney et al., 2000; Hummel et al., 2002; Gow et al., 2004; Nakahara et al., 2004; Yoshida et al., 2006). We have recently shown that air puffs applied to the posterior oropharyngeal wall will elicit 
a sensory evoked potential, termed the PSEP (pharyngeal sensory evoked potential), that is characterized by four component peaks: P1, N1, P2, N2, named for their polarity (positive or negative) and relative order (Wheeler-Hegland et al., in press). These peaks are likely analogous to somatosensory evoked potential (SEP) and respiratory-related evoked potential (RREP) peaks with similar latencies and scalp recording locations. The $\mathrm{P} 1$ peak is related to the initial arrival of a sensory stimulus at the somatosensory cortex, the $\mathrm{N} 1$ and $\mathrm{P} 2$ peaks are associated with attention to and processing of the stimulus and the $\mathrm{N} 2$ peak is thought to be related to sensory inhibition and decision processing for behavioral responses (Logie et al., 1998; Webster and Colrain, 2000; Crowley and Colrain, 2004; Chan and Davenport, 2008; Lijffijt et al., 2009).

The term "gating" refers to the ability of the cerebral cortex to include or exclude sensory information from throughput to cortical areas based on its relative redundancy or relevance. Sensory gating out of information is defined as reduced neural activity occurring with increased stimulus redundancy, measured via the scalp sensory evoked potential (Arnfred et al., 2001). Widely studied with the auditory evoked potential (AEP) using a paired-click paradigm (Adler, 1982; Adler et al., 1998), gating has been studied in other sensory systems, including somatosensation and respiration (Arnfred et al., 2001; Chan and Davenport, 2008). In each of these systems, the pairing of two identical stimuli (S1 and S2) results in reduced amplitude (gating out) of S2 such that the ratio of S2/S1 is between 0.33 and 0.59. The SEP P50/RREP P1 peaks and the SEP N100/RREP N1 peaks (analogous to the PSEP P1 and $\mathrm{N} 1$, respectively), as well as the AEP peaks $\mathrm{P} 1, \mathrm{~N} 1$, and $\mathrm{P} 2$, are all considered "gating" peaks because the S2 elicits much smaller amplitude peaks when the paired stimuli are presented within $500 \mathrm{~ms}$ of each other.

While the pattern generators that integrate sensory information for airway defense behaviors are in the brainstem (Bolser and Davenport, 2002), the cerebral cortex also plays a role in the genesis of these behaviors (Davenport et al., 2002; Gow et al., 2004; Eccles, 2009). As such, the cortical response to afferent information from the pharynx is important. Oropharyngeal sensory input is specifically important for initiating and modifying the motor events comprising a swallow, and there is some evidence to suggest stimulation of this area can induce coughing. Studying sensation and sensory gating is important to our understanding of these airway defense behaviors. Single swallows, often lasting less than $1000 \mathrm{~ms}$, are readily modified with changes to bolus parameters, such as consistency or volume (Ishida et al., 2002; Lawless et al., 2003). This suggests a need for consistent, online sensory monitoring throughout the duration of a swallow. Therefore, if oropharyngeal stimuli were gated out, the likelihood of losing important information related to the bolus viscosity, size, or location may increase, and hence so might the likelihood of a penetration or aspiration event. As such our overall hypothesis is that central neural gating out of sensory information is absent for stimuli applied to the posterior oropharyngeal wall. Stated another way, oropharyngeal sensory stimuli are gated in at the level of the cerebral cortex. In order to test this hypothesis we identified the following specific aims: (1) to record event-related evoked potentials from the posterior oropharyngeal wall (PSEPs) using paired-pulse air puff stimuli delivered $500 \mathrm{~ms}$ apart. It was hypothesized that distinct PSEP waveforms including component peaks $\mathrm{P} 1, \mathrm{~N} 1, \mathrm{P} 2$, and $\mathrm{N} 2$ would exist, for the first (S1) and the second (S2) stimulus, and (2) to compare the amplitude of component peaks P1, N1, P2, and N2, from the S1 and S2 PSEP waveforms. It was hypothesized that no significant differences would exist for the amplitude of the S1 and S2 peaks, and that gating ratios (S2/S1) would be greater than 0.60 for the PSEP component peaks.

\section{MATERIALS AND METHODS}

Twenty-three ( 7 men, 16 women) healthy volunteers participated in the study. The mean age was $20.65 \pm 3.43$ years. All subjects self reported no history of cancer in the head or neck regions, neurologic disease, chronic respiratory disease, or dysphagia. Participants were asked to refrain from caffeine for $12 \mathrm{~h}$ prior to participating in the experiment and were not currently smokers. The experimental procedures were explained to the participants upon arrival to the laboratory, and all participants provided written informed consent to participate. The University of Florida Health Science Center Institutional Review Board approved the study.

\section{PULMONARY FUNCTION TESTING}

All participants were screened with a pulmonary function test to ensure no restrictions or obstructions existed that would indicate airway pathology that would potentially impact sensation. Forced vital capacity (FVC) was measured at least three times for each participant. The participant was instructed to respire normally through a filtered mouthpiece with nose clips in place. Following three to four rest breaths, they were asked to provide a deep inspiration followed by a forced expiration. Instructions were based on the American Thoracic Society standard for spirometry testing (Miller et al., 2005). The forced expired volume within $1 \mathrm{~s}\left(\mathrm{FEV}_{1}\right)$ and the FVC were recorded (Jaeger Toennies, Medizintechnikmit System) and the ratio of $\mathrm{FEV}_{1} / \mathrm{FVC}$ was used for analysis. All subjects had an $\mathrm{FEV}_{1} / \mathrm{FVC}$ ratio greater than $75 \%$ predicted. The respiratory resistance at $5 \mathrm{~Hz}$ (R5) was measured with impulse oscillometry (Jaeger Toennies, Medizintechnikmit System). The mean R5 resistance was $3.00 \pm 0.67 \mathrm{cmH} \mathrm{O}_{2} \mathrm{O} \cdot \mathrm{L}^{-1} \cdot \mathrm{s}$, within the predicted normal range for all participants.

\section{SUBJECT PREPARATION AND PROTOCOL}

A 32-electrode Neuroscan Quickcap ${ }^{\mathrm{TM}}$ based on the International 10-20 system was positioned on the participant's head and connected to the SynAmps ${ }^{2}$ Neuroscan System. Conducting gel was applied through each electrode in order to establish scalp contact and maintain impedance levels below $5 \mathrm{k} \Omega$. Bipolar electrodes were placed on the skin above and below the left eye for recording vertical electro-oculogram (VEOG) activity. Synamps ${ }^{2}$ amplifiers (Neuroscan, El Paso, TX, USA) and SCAN version 4.3 acquisition software (Neuroscan, El Paso, TX, USA) were used to record the EEG signal onto a desktop computer. The sampling rate was set to $1000 \mathrm{~Hz}$ per channel with a recording bandpass of DC to $200 \mathrm{~Hz}$. The EEG activity was referenced to linked earlobes. SCAN version 4.3 analysis software (Neuroscan, El Paso, TX, USA) was used for data analysis.

Participants were instructed to relax and sit comfortably in a chair with the neck, back, and arms supported. A mouthpiece with a polyethylene tube was placed in the mouth, and a flexible laryngoscope 
was inserted through the tube (Figure 1). The laryngoscopic images were displayed, but not recorded, on a computer screen. Both the laryngoscope and computer were components of the JEDMED StroboCAM II ${ }^{\oplus}$ system (JEDMED Instrument Co., St Louis, MO, USA). In this manner, the laryngoscope allowed for visualization and verification of tube placement for air puff delivery. The laryngoscope itself was covered with a hygienic sheath (Slide-On ${ }^{\circledR}$ Sheath for Sensory Testing, Medtronic Xomed, Inc., Jacksonville, FL, USA) that has a small port through which the air puffs were delivered. The port was connected to an air tank connected to a solenoid valve that delivered air puffs to the laryngoscope tube. The air tank was in-series with a digital manometer (Fluke 713 30G, John Fluke MFG Co-Inc, Everett, WA, USA), allowing for control of air puff pressure. A manual trigger system that provided an electrical output was used to initiate the data sample collected by the computer simultaneously with air puff delivery.

Air puffs were delivered in pairs with an inter-stimulus interval (ISI) of $500 \mathrm{~ms}$. This interval was chosen based on the work of Arnfred et al. (2001) that identified $500 \mathrm{~ms}$ as being the optimal paired stimulus interval for measuring sensory gating. Four sets of 50 air puff pairs were delivered, yielding a total of 200 stimuli for stimulus 1 (S1) and stimulus 2 (S2). Between each air puff pair (within sets of 50) there was a minimum of 2-s interval. This took approximately 3-4 min per set; participants rested for at least 2 min between sets and were allowed to drink water at that time, if desired.

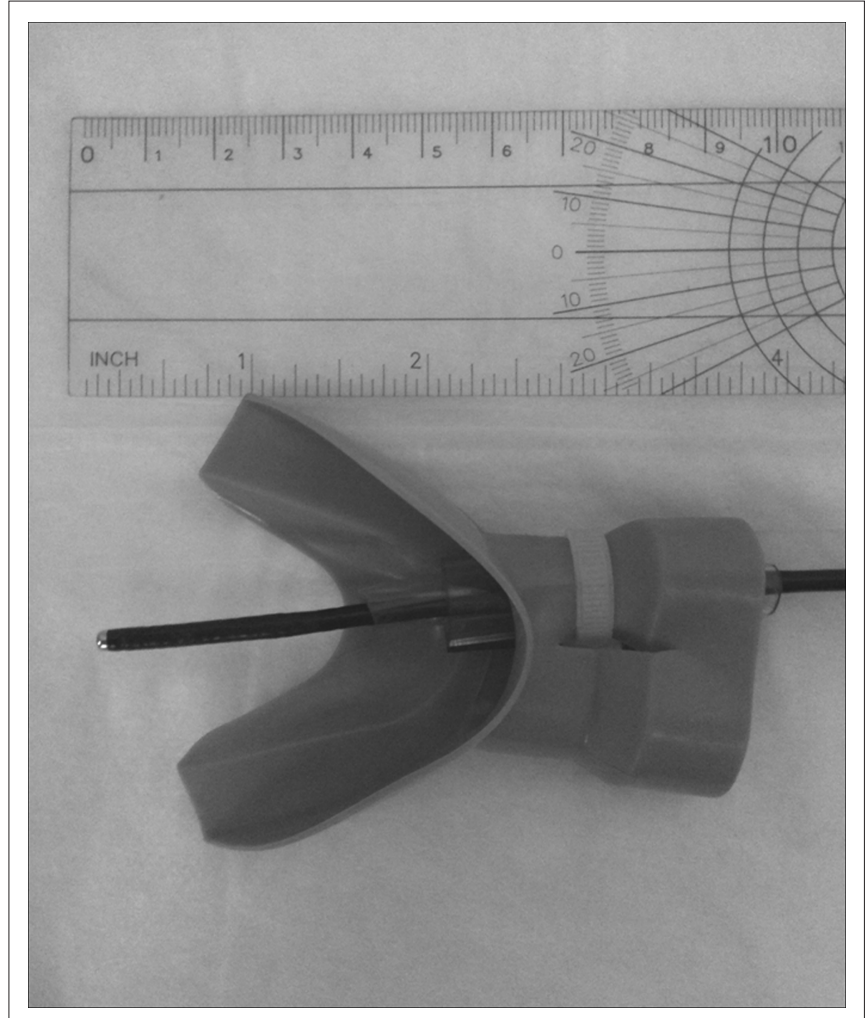

FIGURE 1 | Mouthpiece with polyurethane tubing used for placement of the flexible laryngoscope with the air puff delivery port. The laryngoscope was inserted posteriorly toward the oropharyngeal wall, therefore oriented at an approximately $90^{\circ}$ angle.

\section{EXPERIMENTAL PROTOCOL}

Air puff pairs were delivered through the laryngoscope port, inserted and anchored through the mouthpiece. In order to place the delivery port, participants were instructed to relax their jaw and tongue, and to breathe through the mouth. Participants were then asked to sustain "ah" which raised the soft palate and allowed for insertion of the tube through the posterior oral cavity and into the oropharyngeal region. The delivery port was situated immediately in front of, but not touching, the paramedial posterior oropharyngeal wall, within approximately $5 \mathrm{~mm}$ of midline on either side. Placement was verified visually on the computer screen. Once in place, preliminary air puffs were delivered in order to establish that the participant could feel the air puff specifically on the posterior oropharyngeal wall. It was not acceptable to feel generalized pressure in the throat, and the air puff pressure was adjusted until subjects indicated they felt a discrete air puff stimulus on the posterior oropharyngeal wall. The duration of each air puff pulse was $150 \mathrm{~ms}$; pressure of the air puffs was recorded for each subject, and ranged from 15 to $30 \mathrm{cmH}_{2} \mathrm{O}$ at the air tank. As pressure dissipates as it travels through the tube, the pressure at the delivery port ranged between 1 and $4 \mathrm{cmH}_{2} \mathrm{O}$.

\section{DATA ANALYSIS}

Five-hundred millisecond epochs (100 ms pre-stimulus and $400 \mathrm{~ms}$ post stimulus) were sampled when the air puff stimulus was triggered for both S1 and S2. During offline analysis using SCAN 4.3 software (Neuroscan, El Paso, TX, USA) each S1 and S2 data frame was reviewed and the inclusion criteria for epochs were: (1) no VEOG eyeblink activity, and (2) no change of EEG activity exceeding $50 \mu \mathrm{V}$. Responses to stimuli that were confounded by artifacts were excluded from analysis. A minimum of 190 air puff epochs were averaged for both stimuli to obtain the PSEP waveforms. The peak latencies were measured from the time of S1 and S2 onset, and amplitudes were measured from baseline to peak for each component peak.

Sensory gating is based on peak amplitude and accuracy is dependent upon measurement of the same peak for the S1 and S2 waveform. As such, a latency criterion of \pm 10 ms between the S1 and S2 peaks was established. Identification of the component peaks has been described in detail elsewhere (Wheeler-Hegland et al., in press). Briefly, the averaged S1 and S2 waveforms for each subject were displayed concurrently on a computer screen. As well, along with the waveforms a 2-D head model corresponding temporally to the waveform and depicting all recording electrodes was displayed (Figure 2) using source localization software (Scan 4.3). The software was used to determine EEG amplitude at and between the electrodes. Concurrent visualization of the S1 and S2 waveforms and 2-D head models allowed for identification of the positive and negative component peaks. Once a peak was identified for each stimulus, the recording electrode closest to the area of greatest positive or negative EEG amplitude was identified. This is termed the "hot spot" electrode. If the hot spot electrode was different between S1 and S2 the closest electrode to the hot spot that was common between the two stimuli was chosen.

Descriptive statistics including mean values and standard deviations were used to describe the latency, amplitude, and S2/S1 ratio data. A repeated measures analysis of variance was used to test for significant differences in the amplitude of S2 and S1 responses for each of the four component peaks. 


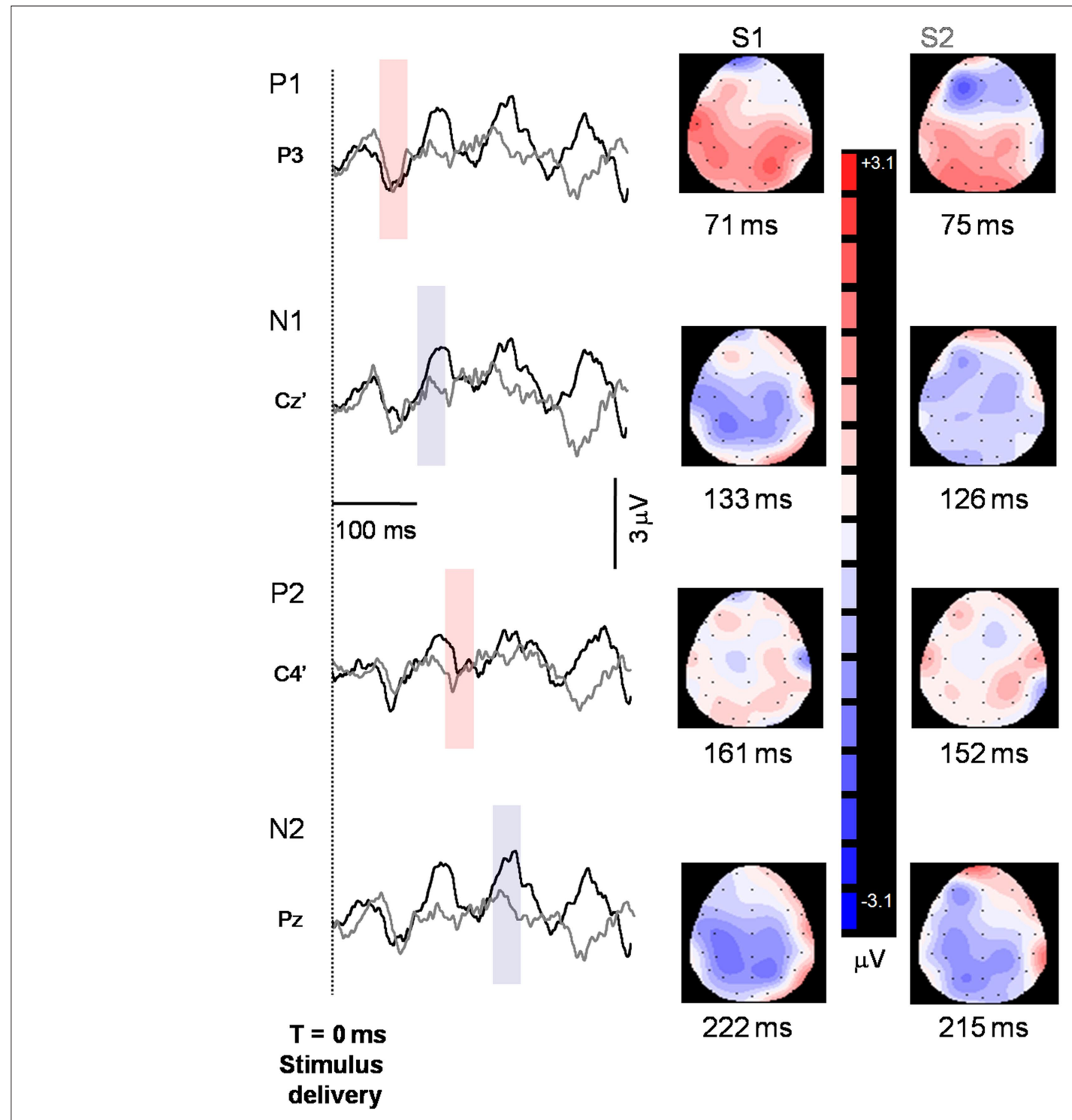

FIGURE 2 | Representative S1 (black tracings) and S2 (gray tracings) peaks identified from one participant. Peaks are indicated to the left of the axis, and the recording electrode is indicated below the peak. Shaded regions indicate where

\section{RESULTS}

Each of the component peaks P1, N1, P2, and N2 were present for both the S1 and S2 stimuli in all subjects. Their mean latencies and amplitudes are in Table 1. Results of repeated measures ANOVA with within subject factors stimulus (S1 or S2) and peak (P1, N1, $\mathrm{P} 2$, N2) showed non-significant differences between the S1 and S2 peak amplitudes $(F=1.428, \mathrm{df}=22.3, p=0.243)$.

Gating ratios were computed by dividing the amplitude of S2 by that of $\mathrm{S} 1$ (S2/S1). A ratio of less than 0.60 was established as criteria for S2 to be considered gated (Chan and Davenport, 2008). Mean gating ratio and standard error for $\mathrm{P} 1$ is $0.92(0.13)$, for $\mathrm{N} 1$ is $1.12(0.11)$, for P2 is 1.09 (0.09), and for N2 is $0.96(0.10)$. Figure 3 depicts the gating ratio of each peak for individual subjects. In total, the $\mathrm{P} 1$ peak was considered gated in 7 of 23 subjects, the $\mathrm{N} 1$ peak was gated in 5 of 23 subjects, and P2 and N2 were each gated in 3 of peaks were identified for the two stimuli and correspond temporally to the 2-D maps on the right side of the figure. Note that downward deflections indicate more positive polarity. Baseline was established from a 100-ms pre-stimulus epoch.

23 subjects. When gating did occur, there was no pattern observed between the peaks. For example, gating of P1 did not necessarily mean that $\mathrm{N} 1$ or other subsequent peaks were also gated.

\section{DISCUSSION}

Pharyngeal sensory evoked potential P1, N1, P2, and N2 peaks were present in response to each stimulus in a paired-air puff paradigm with an ISI of $500 \mathrm{~ms}$. The S2 response failed to yield significantly different amplitude versus the $\mathrm{S} 1$ response amplitude in the $\mathrm{P} 1, \mathrm{~N} 1, \mathrm{P} 2$, or N2 peaks. The air puff parameters for S1 and S2 were constant indicating that these results were not a function of variations in stimulus pressure intensity or duration. Comparison and analysis of the amplitudes of the S1 and S2 peaks shows that repetitive redundant input to the oropharynx is not gated out. These results are inconsistent with those 
previously reported for other sensory modalities, thus supporting our a priori hypothesis that sensory central neural gating out would be absent with identical, paired-pulse air puff stimuli delivered to the oropharynx. These differences are not likely due to protocol deviations as the spacing of stimuli was as recommended by Arnfred et al. (2001) examining the effect on ISI on gating relationships. Their study found $500 \mathrm{~ms}$ ISI to be most effective in terms of the degree of gating present in both somatosensory and auditory modalities. Chan and Davenport (2008) also used $500 \mathrm{~ms}$ ISI and showed gating is present for the RREP and mouth evoked potential recorded with air puffs delivered to the inner surface of the cheek (buccal cavity).

Gating deficits, a term used to indicate a lack of reduction in evoked potential peak amplitude for S2 versus S1 redundant stimuli, exist in various pathologies including some strokes (Staines et al., 2002), schizophrenia (Adler, 1982), and obsessive-compulsive disorder (Nanbu et al., 2010). Gating deficits are hypothesized to indicate abnormal influx of redundant sensory information that impedes the brain's ability to efficiently process sensory input, and may impede higher cognitive processes (Lijffijt et al., 2009). However, it has been shown that healthy people can endogenously modulate gating under certain conditions. For example, directing research participants to attend to specifically to the $\mathrm{S} 2$ stimulus can result in disinhibition of the S2 response (Guterman et al., 1992; Jerger et al., 1992; White and Yee, 1997; Webster and Colrain, 2000). This effect is dependent upon the type of attentional resources required, as simply directing a participant to attend to the presence of stimuli does not affect gating ratios (Harver et al., 1995; Chan and Davenport, 2009). Therefore, it is necessary to require some higher level of processing in order to effectively disinhibit the $\mathrm{S} 2$ response.

Table 1 | Mean values and standard errors (SE) for latency and amplitude of the $\mathbf{S} 1$ and $\mathbf{S} 2$ stimuli.

\begin{tabular}{|c|c|c|c|c|}
\hline & \multicolumn{2}{|c|}{ Latency (ms) Mean (SE) } & \multicolumn{2}{|c|}{ Amplitude $(\mu \mathrm{v})$ Mean (SE) } \\
\hline & s1 & S2 & S1 & S2 \\
\hline P1 & $57.96(2.36)$ & $59.22(2.38)$ & $1.22(0.18)$ & $1.01(0.19)$ \\
\hline N1 & $90.00(3.91)$ & $92.74(3.86)$ & $-1.19(0.17)$ & $-1.20(0.18)$ \\
\hline P2 & $122.00(4.65)$ & $124.26(4.92)$ & $1.03(0.09)$ & $1.06(0.10)$ \\
\hline N2 & $165.13(6.12)$ & $166.13(6.10)$ & $-1.32(0.18)$ & $-1.08(0.10)$ \\
\hline
\end{tabular}

Participants in the current study were not given any instructions on whether to attend to the stimuli, and all watched a movie of their choice during the experiment. However, we have reported for a subpopulation of the participants in the current study there was a significant urge-to-cough during the experiment (WheelerHegland et al., in press). While it was not measured explicitly in the current study, previous research tells us that repetitive air puffs delivered to the posterior faucial arches can induce the urge-toswallow or swallowing (Theurer et al., 2005). Thus, there was likely some degree of generalized attention to the sensations generated via air puff stimuli. Guterman et al. (1992) propose that attention as it relates to gating can serve to facilitate cortical responsiveness to incoming stimuli. Chan and Davenport (2009) demonstrated for both the mouth and respiratory evoked potential that ratios in an "attend" to the stimulus condition were still gated. Hence it is possible that for any stimulus delivered to the oropharyngeal region, whether redundant or novel, there may be a level of attention related to responsiveness for airway defense (e.g., swallowing or cough), it is unlikely that attention to the stimuli accounts for the overall lack of gating found in this study.

Attention should also be given to the degree of variability in amplitude measures and the possibility of type 2 error, or failing to reject the null hypothesis (that no differences would be detected) when significant differences do exist. Typically for studies on sensory gating the primary outcome measure is the S2/S1 (gating) ratio with criterion set at 0.6 or less for a stimulus to be considered gated. The repeated measures ANOVA test was included in this analysis in the case ratios were very close to that ratio criteria there would be some measure of determining whether a significant difference existed between the two stimuli. Therefore, while the possibility of type II error cannot be ruled-out, based on degree to which the gating ratios are greater than 0.6 there is confidence in the conclusion that these are peaks are not gated. To further check this, a one-sample $t$-test was completed for gating ratios ( mean $=1.02$, $\mathrm{SD}=0.52$ ) against $\mathrm{S} 2 / \mathrm{S} 1=0.60$ that yielded a significant difference $(t=7.742, \mathrm{df}=91, p<0.000)$, indicating the mean gating ratio was greater than 0.6 .

Our method of identifying S1 and S2 component peaks is novel in that we did not identify a recording electrode a priori and instead identified the hot spot electrode for each participant as part of data analysis. We developed this method of peak identification because of the availability of 2-D mapping software that allowed for visualization of dipole evolution, from initial creation to the

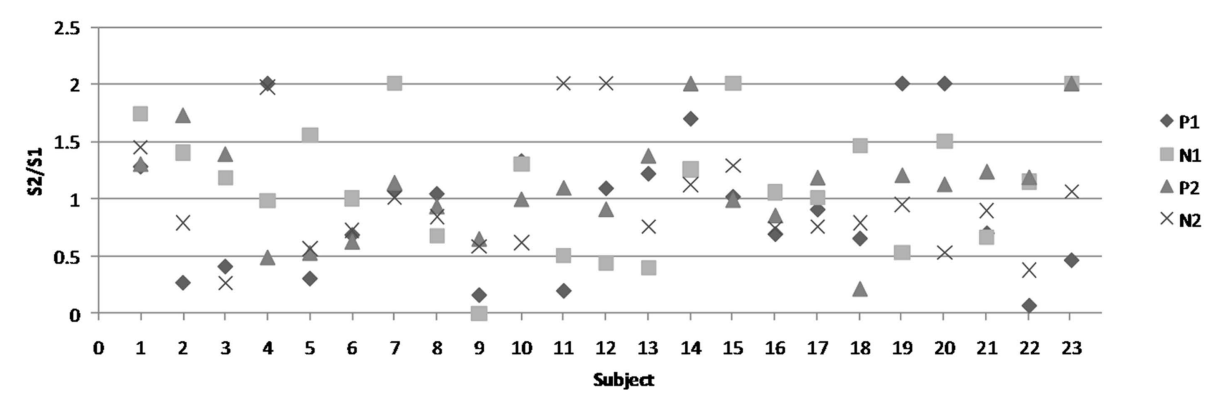

FIGURE 3 | Gating ratios of each peak for all individual subjects. The figure legend is on the right-hand side. Each shape represents one of the four peaks. 
peak. It was therefore possible to identify the strongest recording location within a latency window for each dipole, accounting for individual differences in scalp topography in our analyses. Studies including the Arnfred study, and the Chan and Davenport studies chose a priori recording electrodes and then identified the peaks at those electrodes within a specific latency window. What is ultimately important is that the same recording electrodes were chosen from both the first and second stimulus within the same latency windows, ensuring comparison of the same peak from S1 and S2, which is fundamental to the measurement of sensory gating.

Sensory gating is thought to be the result of an effect for the S1 stimulus on subcortical structures, notably the thalamus (Kaji, 2001), and possibly its connections with the hippocampus and prefrontal cortex (Tregellas et al., 2007), ultimately acting on the throughput of the S2 stimulus. Event-related EEG recording from the scalp cannot determine the precise neuroanatomical substrates due to poor spatial resolution, thus rendering it difficult to make any conclusion regarding the structure(s) that regulate(s) gating of oropharyngeal sensory information. Presently it is only possible to hypothesize based on results from imaging studies. Multiple imaging studies have shown diffuse areas of cortical activation during swallowing (Hamdy et al., 1999; Mosier et al., 1999; Martin et al., 2001; Toogood et al., 2005), swallow-related behaviors (Martin et al., 2004; Lowell et al., 2008; Malandraki et al., 2009), coughing (Simonyan et al., 2007), urge-to-cough (Mazzone et al., 2007), and oral air puff stimulation (Lowell et al., 2008; Sörös et al., 2008). These findings provide several possible neuroanatomical substrates for the findings of the current study. Operating under the hypothesis that the thalamus is a key structure for sensory gating, it is

\section{REFERENCES}

Addington, W. R., Stephens, R. E., Gilliland, K., and Rodriguez, M. (1999). Assessing the laryngeal cough reflex and the risk of developing pneumonia after stroke. Arch. Phys. Med. Rehabil. 80, 150-154.

Adler, L. E. (1982). Neurophysiological evidence for a defect in neuronal mechanisms involved in sensory gating in schizophrenia. Biol. Psychiatry 17, 639-654.

Adler, L. E., Olincy, A., Waldo, M., Harris, J. G., Griffith, J., Stevens, K., Flach, K., Nagamoto, H., Bickford, P., and Leonard, S. (1998). Schizophrenia, sensory gating, and nicotinic receptors. Schizophr. Bull. 24, 189-202.

Arnfred, S. M., Eder, D. N., Hemmingsen, R.P., Glenthøj, B.Y., and Chen, A. C. N. (2001). Gating of the vertex somatosensory and auditory evoked potential P50 and the correlation to skin conductance orienting response in healthy men. Psychiatry Res.101, 221-235.

Aviv, J. E., Sacco, R. L., Mohr, J., Thompson, J., Levin, B., Sunshine, S., Thomson, J., and Close, L. G. (1997). Laryngopharyngeal sensory testing with modified barium swallow as predictors of aspiration pneumonia after stroke. Laryngoscope 107, 1254-1260.
Bolser, D. C., and Davenport, P.W. (2002). Functional organization of the central cough generation mechanism. Pulm. Pharmacol. Ther. 15, 221-225.

Boushey, H. A., Richardson, P. S., Widdicombe, J. G., and Wise, J. C. (1974). The response of laryngeal afferent fibres to mechanical and chemical stimuli. J. Physiol. 240, 153-175.

Bradley, R. M. (2000). Sensory receptors of the larynx. Am. J. Med. 108(Suppl. 4a), 47S-50S.

Chan, P.Y.S., and Davenport, P.W. (2008). Respiratory-related evoked potential measures of respiratory sensory gating. J. Appl. Physiol. 105, 1106.

Chan, P. Y. S., and Davenport, P. W. (2009). Respiratory-related-evoked potential measures of respiratory sensory gating in attend and ignore 438-445.

Crowley, K. E., and Colrain, I. M. (2004). A review of the evidence for $\mathrm{P} 2$ being an independent component process: age, sleep and modality. Clin. Neurophysiol. 115, 732-744.

Davenport, P., Sapienza, C., and Bolser, D. (2002). Psychophysical assessment of the urge-to-cough. Eur. Respir. Rev. 12, 249-253. conditions. J. Clin. Neurophysiol. 26,

interesting that several thalamic nuclei are active during swallowing coughing, and oral air puff stimulation. Consider as well the functional cortical model of swallowing substrates proposed by Mosier and Bereznaya (2001), consisting of five modules and two parallel loops. Within their model is the IFG-S2-CC-BGTHAL module consisting of the inferior frontal gyrus, secondary sensory cortex, corpus callosum, and basal ganglia and thalamus. This group of structures is thought to integrate sensory information about the bolus with some internal representation of swallowing movement. It would therefore fit the functionality of this module and with the thalamus gating hypothesis if gating in or out occurred as part of the decision-making process. Future studies incorporating EEG and functional magnetic imaging, or magnetoencephalography would enable anatomical correlation and electrophysiological data to be collected simultaneously in order to glean more specific information regarding the anatomy and physiology of oropharyngeal sensory gating.

For some patients with dysphagia (disordered swallowing) or dystussia (disordered cough) gating out of oropharyngeal sensory information may be related to dysfunctional formation or modulation of the motor output plan. For example, if during the course of a meal, pharyngeal sensory information is gated out at a level that does not allow for normal initiation of swallow motor output, perhaps a progressive delay in swallow initiation would occur and result in airway compromise. This is highly speculative, and future research should examine oropharyngeal sensory gating in disordered populations in order to ascertain: (1) pharyngeal gating characteristics, and (2) potential relationship to swallow dysfunction and airway compromise.

Doty, R. W. (1951). Influence of stimulus pattern on reflex deglutition. Am. J. Physiol. 166, 142-158.

Eccles, R. (2009). Central mechanisms IV: conscious control of cough and the placebo effect. Handb. Exp. Pharmacol. 187, 241-262.

Fujiu, M., Toleikis, J. R., Logemann, J. A., and Larson, C. R. (1994). Glossopharyngeal evoked potentials in normal subjects following mechanical stimulation of the anterior faucial pillar. Electroencephalogr. Clin. Neurophysiol. 92, 183-195.

Gow, D., Hobson, A. R., Furlong, P., and Hamdy, S. (2004). Characterising the central mechanisms of sensory modulation in human swallowing motor cortex. Clin. Neurophysiol. 115, 2382-2390.

Guterman, Y., Josiassen, R.C., and Bashore, T. R. Jr. (1992). Attentional influence on the P50 component of the auditory event-related brain potential. Int. J. Psychophysiol. 12, 197-209.

Hamdy, S., Mikulis, D. J., Crawley, A., Xue, S., Lau, H., Henry, S., and Diamant, N. E. (1999). Cortical activation during human volitional swallowing: an event-related fMRI study. Am. J. Physiol. 277(Pt 1), G219-G225.

Harver, A., Squires, N. K., Bloch-Salisbury, E., and Katkin, E. S. (1995). Event- related potentials to airway occlusion in young and old subjects. Psychophysiology 32, 121-129.

Hummel, T., Haenel, T., and Hull, D. (2002). Assessment of pharyngeal sensitivity to mechanical stimuli using psychophysical and electrophysiological techniques. Pulm. Pharmacol. Ther. 15, 321-325.

Hwang, J. C., St John, W. M., and Bartlett, D. Jr. (1984). Receptors responding to changes in upper airway pressure. Respir. Physiol. 55, 355-366.

Ishida, R., Palmer, J. B., and Hiiemae, K. M. (2002). Hyoid motion during swallowing: factors affecting forward and upward displacement. Dysphagia 17, 262-272.

Jerger, K., Biggins, C., and Fein, G. (1992). P50 suppression is not affected by attentional manipulations. Biol. Psychiatry 31, 365-377.

Kaji, R. (2001). Basal ganglia as a sensory gating devise for motor control. J. Med. Invest. 48, 142-146.

Kitagawa, J., Nakagawa, K., Hasegawa, M., Iwakami, T., Shingai, T., Yamada, Y., and Iwata, K. (2009). Facilitation of reflex swallowing from the pharynx and larynx. J. Oral. Sci. 51, 167-171.

Kitagawa, J. I., Shingai, T., Takahashi, Y., and Yamada, Y. (2002). Pharyngeal 
branch of the glossopharyngeal nerve plays a major role in reflex swallowing from the pharynx. Am. J. Physiol. Regul. Integr. Comp. Physiol. 282, 1342-1347.

Lawless, H. T., Bender, S., Oman, C., and Pelletier, C. (2003). Gender, age, vessel size, cup vs. straw sipping, and sequence effects on sip volume. Dysphagia 18, 196-202.

Lijffijt, M., Lane, S. D., Meier, S. L., Boutros, N. N., Burroughs, S., Steinberg, J. L., Moeller F. G., and Swann, A. C. (2009). P50, N100, and P200 sensory gating: relationships with behavioral inhibition, attention, and working memory. Psychophysiology 46, 1059-1068.

Logie, S. T., Colrain, I. M., and Webster, K. E. (1998). Source dipole analysis of the early components of the RREP. Brain Topogr. 11, 153-164.

Lowell, S. Y., Poletto, C. J., Knorr-Chung, B. R., Reynolds, R. C., Simonyan, K., and Ludlow, C. L. (2008). Sensory stimulation activates both motor and sensory components of the swallowing system. Neuroimage 42, 285-295.

Malandraki, G. A., Sutton, B. P., Perlman, A. L., Karampinos, D. C., and Conway, C. (2009). Neural activation of swallowing and swallowing-related tasks in healthy young adults: an attempt to separate the components of deglutition. Hum. Brain Mapp. 30, 3209-3226.

Maloney, S., Bell, W., Shoaf, S., Blair, D., Bastings, E., Good, D., and Quinlivan, L. (2000). Measurement of lingual and palatine somatosensory evoked potentials. Clin. Neurophysiol. 111, 291-296.

Martin, R. E., Goodyear, B. G., Gati, J. S., and Menon, R. S. (2001). Cerebral cortical representation of automatic and volitional swallowing in humans. J. Neurophysiol. 85, 938-950.

Martin, R. E., MacIntosh, B. J., Smith, R. C., Barr, A. M., Stevens, T. K., Gati, J. S., and Menon, R. S. (2004). Cerebral areas processing swallowing and tongue movement are overlapping but distinct: a functional magnetic resonance imaging study. J. Neurophysiol. 92, 2428-2443.
Mazzone, S. B., McLennan, L., McGovern, A. E., Egan, G. F., and Farrell, M. J. (2007). Representation of capsaicinevoked urge-to-cough in the human brain using functional magnetic resonance imaging. Am. J. Respir. Crit. Care Med. 176, 327-332.

Miller, M. R., Hankinson, J., Brusasco, V. Burgos, F., Casaburi, R., Coates, A., Crapo, R., Enright, P., van der Grinten, C. P., Gustafsson, P., Jensen, R., Johnson, D.C., MacIntyre, N.,McKay, R., Navajas, D., Pedersen, O. F., Pellegrino, R., Viegi, G., Wanger, J., and ATS/ERS Task Force. (2005). Standardisation of spirometry. Eur. Respir. J. 26, 319-338.

Mori, M., and Sakai, Y. (1972). Re-examination of centrally-induced cough in cats using a micro-stimulation technique. Jpn. J. Pharmacol. 22, 635-643.

Mosier, K., and Bereznaya, I. (2001). Parallel cortical networks for volitional control of swallowing in humans. Exp. Brain Res. 140, 280-289.

Mosier, K., Patel, R., Liu, W. C., Kalnin, A., Maldjian, J., and Baredes, S. (1999). Cortical representation of swallowing in normal adults: functional implications. Laryngoscope 109, 1417-1423.

Mu, L., and Sanders, I. (2000). Sensory nerve supply of the human oro- and laryngopharynx: a preliminary study. Anat. Rec. 258, 406.

Nakahara, H., Nakasato, N., Kanno, A., Murayama, S., Hatanaka, K., Itoh, H., and Yoshimoto, T. (2004). Somatosensory-evoked fields for gingiva, lip, and tongue. J. Dent. Res. 83, 307.

Nanbu, M., Kurayama, T., Nakazawa, K., Matsuzawa, D., Komiya, Z., Haraguchi, T., Ogura, H., Hashimoto, T., Yoshida, S., Iyo, M., and Shimizu, E. (2010). Impaired P50 suppression in fear extinction in obsessive-compulsive disorder. Prog. Neuropsychopharmacol. Biol. Psychiatry 34, 317-322.

Sant'Ambrogio, G., BrambillaSant'Ambrogio, F., and Mathew, O. P. (1986). Effect of cold air on laryngeal mechanoreceptors in the dog. Respir. Physiol. 64, 45-56.
Sciortino, K., Liss, J. M., Case, J. L. Gerritsen, K. G., and Katz, R. C. (2003). Effects of mechanical, cold, gustatory, and combined stimulation to the human anterior faucial pillars. Dysphagia 18, 16-26.

Simonyan, K., Saad, Z. S., Loucks, T. M. Poletto, C. J., and Ludlow, C. L. (2007) Functional neuroanatomy of human voluntary cough and sniff production. Neuroimage 37, 401-409.

Smith Hammond, C. A., Goldstein, L. B., Zajac, D. J., Gray, L., Davenport, P. W. and Bolser, D.C. (2001). Assessment of aspiration risk in stroke patients with quantification of voluntary cough. Neurology 56, 502-506.

Sörös, P., Lalone, E., Smith, R., Stevens, T., Theurer, J., Menon, R., and Martin, R. (2008). Functional MRI of oropharyngeal air-pulse stimulation. Neuroscience 153, 1300-1308.

Staines, W. R., Black, S. E., Graham, S. J., and McIlroy, W. E. (2002) Somatosensory gating and recovery from stroke involving the thalamus. Stroke 33, 2642-2651.

Storey, A. T. (1968). Laryngeal initiation of swallowing. Exp. Neurol. 20, 359-365.

Teismann, I. K., Steinstraeter, O., Stoeckigt, K., Suntrup, S., Wollbrink, A., Pantev, C., and Dziewas, R. (2007). Functional oropharyngeal sensory disruption interferes with the cortical control of swallowing. BMC Neurosci. 8, 62. doi: 10.1186/1471-2202-8-62

Theurer, J. A., Bihari, F., Barr, A. M., and Martin, R. E. (2005). Oropharyngeal stimulation with air-pulse trains increases swallowing frequency in healthy adults. Dysphagia 20, 254-260.

Toogood, J. A., Barr, A. M., Stevens, T. K. Gati, J. S., Menon, R. S., and Martin, R. E. (2005). Discrete functional contributions of cerebral cortical foci in voluntary swallowing: a functional magnetic resonance imaging (fMRI) "go, no-go" study. Exp. Brain Res. 161, 81-90.

Tregellas, J. R., Davalos, D. B., Rojas, D C., Waldo, M. C., Gibson, L., Wylie,
K., Du, Y. P., and Freedman, R. (2007). Increased hemodynamic response in the hippocampus, thalamus and prefrontal cortex during abnormal sensory gating in schizophrenia. Schizophr. Res. 92, 262-272.

Webster, K. E., and Colrain, I. M. (2000). The respiratory-related evoked potential: effects of attention and occlusion duration. Psychophysiology 37, 310-318.

Wheeler-Hegland, K. M., Pitts, T., Bolser, D. C., and Davenport, P. (in press). Urge to cough with voluntary suppression following mechanical pharyngeal stimulation. Bratslavia Med. J.

White, P.M., and Yee, C. M. (1997). Effects of attentional and stressor manipulations on the P50 gating response. Psychophysiology 34, 703-711.

Yoshida, K., Maezawa, H., Nagamine, T., Fukuyama, H., Murakami, K., and Iizuka, T. (2006). Somatosensory evoked magnetic fields to air-puff stimulation on the soft palate. Neurosci. Res. 55, 116-122.

Conflict of Interest Statement: The authors declare that the research was conducted in the absence of any commercial or financial relationships that could be construed as a potential conflict of interest.

Received: 19 August 2010; accepted: 16 December 2010; published online: 25 January 2011

Citation: Wheeler-Hegland K, Pitts T and Davenport PW (2011) Cortical gating of oropharyngeal sensory stimuli. Front. Physio. 1:167. doi: 10.3389/ fphys.2010.00167

This article was submitted to Frontiers in Respiratory Physiology, a specialty of Frontiers in Physiology.

Copyright (c) 2011 Wheeler-Hegland, Pitts and Davenport. This is an open-access article subject to an exclusive license agreement between the authors and Frontiers Media $S A$, which permits unrestricted use, distribution, and reproduction in any medium, provided the original authors and source are credited. 